ISSN 1794-9831

E-ISSN 2322-7028

Vol. 14 No. 1

Ene - Jun 2017

Cúcuta, Colombia

Recibido:

31 de Mayo de

2016

Aprobado:

31 de Octubre de 2016

* Enfermera.

Candidata a

Magister de

Enfermería

con Énfasis en

Cuidado para Salud

Cardiovascular.

Líder de

Investigaciones

Instituto

Neumológico

del Oriente.

Bucaramanga,

Colombia. Correo

electrónico:

mkrinconr@unal.

edu.co

** Enfermera.

Especialista en

Cuidado Crítico,

Magíster en

Enfermería con

Énfasis en Gerencia

en Salud. Docente

Investigadora.

Universidad de

Santander (UDES).

Bucaramanga,

Colombia. Correo

electrónico:

claudiaconsuelo@

yahoo.co

*** Enfermera

Especialista en

Salud Familiar,

Magíster en

Enfermería.

Profesora Auxiliar.

Universidad

Nacional de

Colombia. Bogotá

D.C., Colombia.

Correo electrónico:

kacorredorp@unal.

edu.co

**** Descriptores

en Ciencias de la

Salud (DeCS), en

la página http://

decs.bvs.br/E/

homepagee.htm de

la Biblioteca

Virtual en Salud de proyecto BIREME,

de la Organización

Mundial de la Salud

y de la Organización

Panamericana de la

Salud.

\section{Adherencia terapéutica en personas con diabetes mellitus tipo $2^{\dagger}$}

\author{
Mayerli Katherine Rincón-Romero* \\ Claudia Torres-Contreras ${ }^{* *}$ \\ Katya Anyud Corredor-Pardo***
}

\title{
RESUMEN
}

Objetivo: determinar la adherencia terapéutica en personas con diabetes mellitus tipo 2 que asisten a una institución de salud, a través de la etiqueta Conducta terapéutica: enfermedad o lesión 1609 presentes. Materiales y Métodos: se realizó un estudio descriptivo de corte transversal con enfoque cuantitativo. La población de estudio estuvo conformada por 178 pacientes que cumplieron con los criterios de selección. Se obtuvo la caracterización sociodemográfica, funcionalidad y estado mental; se midió la adherencia terapéutica en escala de 1 menos adherente y 5 más adherente, con un instrumento validado basado en la etiqueta de resultado del Nursing Outcomes Classification de personas con diabetes mellitus tipo 2. Resultados: el 37.1\% de la población objeto de estudio era de sexo masculino, la ocupación predominante fue la dedicación al hogar, el principal nivel de escolaridad fue primaria, perteneciente en su mayoría a la religión católica, con una media de 11.5 de años de diagnóstico de diabetes tipo 2 y la adherencia terapéutica para la muestra estudiada mostró una media de 3,68. Conclusiones: en la muestra analizada de personas con diabetes tipo 2 se encontró una adherencia terapéutica entre en ocasiones manifestada y con frecuencia manifestada. El incumplimiento de actividad física prescrita es el factor que presentó mayores cifras de no adherencia.

PALABRAS CLAVES: conductas saludables, cumplimiento de la medicación, diabetes mellitus tipo 2, enfermería.

Para citar este artículo / Para citar este artigo/ To reference this article /

Rincón-Romero MK, Torres-Contreras C, Corredor-Pardo KA. Adherencia terapéutica en personas con diabetes mellitus tipo 2. Rev. cienc. cuidad. 2017; 14(1): 40-59.

$\dagger$ Este artículo está basado en la tesis para optar al título de Magister de Enfermería, titulada Autoeficacia y Adherencia Terapéutica en persona con Diabetes Mellitus Tipo 2, 2016. Universidad Nacional de Colombia. 


\section{Therapeutic adherence for people with diabetes mellitus type 2}

\section{ABSTRACT}

Objective: To determine the therapeutic adherence in people with diabetes mellitus type 2 that attend a health institution, through the therapeutic conduct protocol: disease or injury 1609 present. Materials and Methods: a cross-sectional descriptive study with a quantitative focus was performed. The population of the study was made up of 178 patients that met the criteria of selection. The socio-demographic characterization, functionality, and mental state were obtained; the therapeutic adherence was measured on a scale from 1 (less adherent) to 5 (most adherent), with a validated instrument based on the results protocol from Nursing Outcomes Classification for people with diabetes mellitus type 2 . Results: $37,1 \%$ of the study target population were male, the predominant occupation was home-work, the main education level was primary school, mostly from catholic religion, with a mean of 11.5 years with diagnosis of diabetes type 2 and the therapeutic adherence for the studied sample showed a mean of 3,68 Conclusions: in the analyzed sample of people with diabetes type 2 a therapeutic adherence was found between occasionally manifested and frequently manifested. Failure of compliance of prescribed physical activity is the factor that represents higher numbers of non-adherence.

KEYWORDS: medication adherence, health behavior, diabetes mellitus type 2, nursing. 
ISSN 1794-9831

E-ISSN 2322-7028 Vol. 14 No. 1

Ene - Jun 2017

Cúcuta, Colombia

\section{Aderência terapêutica em pessoas com diabetes mellitus tipo 2}

\section{RESUMO}

Objetivo: determinar a aderência terapêutica em personas com diabetes mellitus tipo 2 que assistem a uma instituição da rede de saúde, através da etiqueta Conduta terapêutica: doença ou lesão 1.609 presentes. Materiais e Métodos: realizou-se um estudo descritivo de corte transversal com abordagem quantitativa. A população do estudo esteve conformada por 178 pacientes que cumpriram com os critérios de seleção. Obteve-se a caracterização sócio-demográfica, funcionalidade e estado mental; mediu-se a aderência terapêutica em escala de "1" menos aderente e "5" mais aderente, com um instrumento validado baseado na etiqueta de resultado do Nursing Outcomes Classification de pessoas com diabetes mellitus tipo 2. Resultados: o 37,1 \% da população objeto de estudo era de sexo masculino, a ocupação predominante foi a dedicação ao lar, o principal nível de escolaridade foi educação primária, a maioria pertencente à religião católica, com uma idade média de 11,5 anos de diagnóstico de diabetes tipo 2 e a aderência terapêutica para a amostragem estudada mostrou uma média de 3,68. Conclusões: na amostra analisada de pessoas com diabetes tipo 2 se encontrou uma aderência terapêutica: em ocasiões manifestada e com frequência manifestada. $\mathrm{O}$ incumprimento da atividade física prescrita é o fator que apresentou maiores cifras de não aderência.

PALAVRAS-CHAVE: comportamentos saudáveis, adesão à medicação, diabetes mellitus tipo 2, enfermagem. 


\section{INTRODUCCIÓN}

$\mathrm{E}$ 1 adecuado control metabólico y del perfil glucémico de las personas con diabetes mellitus tipo 2 (DM2) es el objetivo en que se enfocan los programas y políticas del Sistema General de Seguridad Social en Salud (SGSS), siendo una de estas acciones la promoción de la salud y la prevención de riesgo cardiovascular (1). "Estos programas, en el paciente diabético, están dirigidos a inducir y mantener en el tiempo una serie de cambios comportamentales para sostener unos niveles adecuados de glicemia que se acerquen en lo posible a la normalidad, y de esta manera logren modificar de forma importante la historia natural de la enfermedad" (2).

La DM2 es una enfermedad crónica no trasmisible, la cual repercute no sólo sobre la calidad de vida del individuo y su familia, sino que además impacta social y económicamente a los servicios de salud y la sociedad en general (3). A nivel mundial, se estima un incremento en la prevalencia de diabetes de 285 millones $(6,4 \%)$ en el año 2010 a casi 439 millones $(7,7 \%)$ en el año 2030; en Latinoamérica, que incluye 21 países, con casi 500 millones de habitantes y se espera un aumento del $14 \%$ en los próximos diez años, mucho más de lo esperado por el simple crecimiento poblacional (4).

De otro lado, las previsiones de la Organización Mundial de la Salud (OMS) para el año 2030 son de 366 millones de personas con diabetes en todo el mundo y señalan una dimensión de auténtica epidemia, que es el doble de frecuente en las personas de nivel social más bajo respecto a los de nivel más elevado (5). De esta manera, dentro del panorama epidemiológico de la DM2, es preocupante que esta enfermedad se sitúe en segundo lugar como factor de riesgo causante de muerte por enfermedades cardiovasculares (6), asunto que implica la necesidad de acciones para modificar esta situación.

En Colombia, específicamente en el Departamento de Santander, para el año 2015, según datos del Observatorio de Salud Pública de Santander (OSPS) (7), de cada 100 santandereanos 6 tienen diagnóstico de DM2, siendo más prevalente en el grupo de edad de 45 a 64 años, donde 13 de cada 100 personas tiene DM2 y, en relación con el género, es el doble de frecuente en mujeres en comparación con los hombres. También se registra un incremento de la prevalencia en los últimos cinco años, siendo en el 2010 de 5,7\% comparada con las cifras del
2015 de $6,5 \%$. Para visualizar la magnitud del problema es necesario advertir que 9 de cada 100 atenciones realizadas a adultos mayores de 60 años, en el 2013, fueron generadas por causa de la diabetes mellitus, según resultados preliminares reportados por el OSPS derivados del estudio Factores de riesgo para enfermedades crónicas en Santander STEPwise-2015 (7).

Este panorama muestra una situación compleja de gran importancia para la salud pública y para la disciplina de enfermería, porque involucra las situaciones de la vida de la persona sobre las cuales se puede emprender acciones de cuidado para favorecer la experiencia de vivir con una enfermedad crónica de afectación sistémica, a nivel tanto micro como macro vascular, que genera complicaciones severas, e incluso la muerte, cuando no se logra su control y la adherencia al tratamiento farmacológico y no farmacológico (8).

La OMS (5) define el concepto de adherencia como: "el grado en que el comportamiento de una persona -tomar el medicamento, seguir un régimen alimentario y ejecutar cambios del modo de vida- se corresponde con las recomendaciones acordadas de un prestador de asistencia sanitaria". En este sentido, en los países desarrollados, el promedio de la adherencia a los tratamientos a largo plazo alcanza sólo el 50\%, siendo incluso menor en los países en vías de desarrollo" (9), lo que denota la falta de logro de las metas de los programas en los que se busca fomentar dicha adherencia.

Por lo tanto, es necesario realizar un abordaje a nivel de atención primaria para evaluar la adherencia terapéutica de personas con DM2, en el panorama local, y de esta manera identificar factores de riesgo que, a futuro, permitan servir como punto de partida para implementar intervenciones en personas con diabetes tipo 2. Este manejo implica la adopción de estilos de vida saludables y sostenibles en el tiempo como parte fundamental del tratamiento; por lo tanto, una persona con DM2 debe contar con la capacidad de adaptación a la situación de salud que la llevará a adquirir habilidades y competencia en el control de la enfermedad. Para lograrlo, es fundamental la participación activa y voluntaria del paciente con DM2 en el control de la enfermedad (10).

Según el Atlas de la International Diabetes Federation (11), se estima que Colombia gasta, en el mantenimiento de la salud de una persona con diabetes, un promedio de 326 dólares que corresponden a costo 
ISSN 1794-9831

E-ISSN 2322-7028

Vol. 14 No. 1

Ene - Jun 2017

Cúcuta, Colombia directo, lo que equivale a un $4,4 \%$ de lo que se gasta en Estados Unidos y a un 14,3\% de lo que se gasta en España. Los costos directos e indirectos del gasto, según el curso de la enfermedad, se distribuyen de la siguiente manera: un $47 \%$ por el tratamiento de la diabetes (medicamentos), un $24 \%$ por enfermedades cardiacas y coronarias, un $15 \%$ por accidentes cerebrovasculares, un $9 \%$ por amputaciones, un $3 \%$ por nefropatías y un $2 \%$ por retinopatías. Las complicaciones macrovasculares constituyeron el $86 \%$ de los costos directos anuales y el $95 \%$ de los indirectos (11). Lo anterior permite reflexionar acerca de si una mejora en la adherencia terapéutica, previo estudio y conocimiento de la misma, redundaría en una disminución de los costos y de la carga de la enfermedad para estos pacientes.

El fenómeno de adherencia al tratamiento de DM2 en estudios nacionales (12-13) se ha descrito con el fin de determinar su eficiencia y contribuir a la mejora de la calidad de vida de las personas. La adherencia constituye "un tema multifactorial y complejo, en el que se mezclan factores psicológicos, demográficos y de comportamiento, así como aspectos propios debidos a la cronicidad de la afectación y otros que atañen al individuo como tal y como ser social y cultural" (13).

Son escasas las publicaciones que describen los componentes, los hallazgos y los logros de programas o modelos de atención dirigidos a los pacientes con $\mathrm{DM}$ en el escenario colombiano; entendiendo un modelo de atención como un proceso en el cual interactúan los diferentes componentes del servicio a lo largo de la atención en salud y con el paciente como partícipe de tal proceso, con el fin de obtener los mejores resultados con los recursos disponibles (14). Ninguna de las intervenciones descritas en las publicaciones identificadas en el desarrollo de este articulo correspondió a un modelo de atención para DM implementado en Colombia.

Varios escenarios merecen ser explorados para mejorar los programas existentes y construir modelos de atención para el cuidado integral e interdisciplinario de las personas con DM2. Tales escenarios deben trascender la actividad clínica a nivel hospitalario e involucran a pacientes, familia, aseguradores, prestadores, comunidad $\mathrm{y}$ tomadores de decisiones (14).

Según Otero et al. (15) y Gómez et al. (16), en términos generales, un modelo de atención integral para DM2 se debe conducir en correspondencia con cinco estrategias clave para la reducción de la carga que representa la DM: reducción de los factores de riesgo modificables, tamizaje, mejoramiento del diagnóstico y del control glicémico en quienes ya tienen DM, mejoramiento del manejo de las complicaciones microvasculares y mejoramiento del manejo precoz de los riesgos cardiovasculares asociados, para prevenir o retrasar la progresión hacia las complicaciones crónicas.

En Santander, la Secretaria de Salud es la encargada de liderar y garantizar, en nombre del estado colombiano, el acceso a los servicios de promoción y recuperación de la salud como componentes del bienestar, la seguridad y el desarrollo integral de la población del departamento (17). Las políticas gubernamentales están enfocadas hacia la promoción de programas de adulto con enfermedad crónica, donde se aplican las guías de promoción de la salud y prevención de enfermedades en la salud pública, específicamente para la atención de la diabetes mellitus tipo 2. Esto se hace tanto a nivel de la red de salud pública como de la privada (17).

Partiendo de la alta prevalencia de la DM2 en Colombia y Santander (14), se hace necesaria la implementación de estrategias que permitan alcanzar indicadores de mejoría en la adherencia al tratamiento y definir instrumentos de medición de adherencia al tratamiento que puedan apoyar la toma de decisiones en las intervenciones, la comparación entre programas y periodos de tiempo, entre otros aspectos, que favorezcan el éxito de los pacientes con las metas terapéuticas, las cuales se traducirán en mejor calidad de vida.

La presente investigación busca determinar la adherencia terapéutica de individuos con DM2 de un grupo de personas pertenecientes a un programa de riesgo cardiovascular del municipio de Bucaramanga y reconocer los posibles componentes susceptibles de intervención por enfermería.

\section{MATERIALES Y MÉTODOS}

Se realizó un estudio de abordaje cuantitativo, descriptivo, de corte transversal, en el que participaron personas con diagnóstico de diabetes tipo 2 que asistieron a consulta médica de control ambulatorio durante los meses de enero a abril de 2016 y que pertenecían al Programa de Prevención de Riesgo Cardiovascular en una institución de salud, que aceptaron participar de forma voluntaria, firmaron 
el consentimiento informado escrito $\mathrm{y}$, además, cumplieron con los siguientes criterios de inclusión: edad mayor o igual a 18 años y estar diagnosticados con diabetes mellitus tipo 2 en un periodo mayor o igual a 1 año. Se excluyeron personas con alguna alteración de tipo cognitivo o mental que no lograran comprender los instrumentos.

La muestra seleccionada fue aleatoria, no probalística, teniendo en cuenta criterios de inclusión y exclusión en la medida que se respetó el azar de ingreso a la institución. El cálculo de muestra se realizó teniendo en cuenta un universo de 350 pacientes, que fue el promedio de los que asistieron en el último semestre de 2015 al Programa de Prevención de Riesgo Cardiovascular; se tuvo en cuenta un margen de error del $5 \%$ y un nivel de confianza del $95 \%$, obteniendo una muestra de 176 pacientes. Al final del reclutamiento se incluyeron 178 personas que cumplieron los criterios de selección.

La fórmula utilizada para el cálculo del tamaño de la muestra de este estudio fue la siguiente:

$$
n=[\operatorname{EDFF} * \operatorname{Np}(1-\mathrm{p})] /\left[\left(\mathrm{d}^{2} / \mathrm{Z}^{2}{ }_{1-\alpha / 2} *(\mathrm{~N}-1)+\mathrm{p} *(1-\mathrm{p})\right]\right.
$$

donde:

Tamaño de la población (para el factor de corrección de la población finita o fcp) $(N)=350$

Frecuencia \% hipotética del resultado en la población $(p)=65 \%+/-$

Límites de confianza como \% de 100 (absoluto +/-\%) (d) $=5 \%$

Efecto de diseño (para encuestas en grupo - EDFF $)=1$ $\mathrm{Z}=$ valor crítico de la distribución normal estandarizada necesario para construir un intervalo de confianza para la distribución. Un nivel de confianza del $95 \%$ lleva a un valor $\mathrm{Z}$ de 1,96.

\section{Tamaño muestral $(n)$ según el nivel de confianza}

Intervalo Confianza (\%) Tamaño de la muestra $95 \%$ 176

Aplicando la fórmula:

$$
\begin{gathered}
n=[1 * 22,750(1-65)] /\left[\left(5^{2} / 1,96^{2}{ }_{1-\alpha / 2} *(350-1)+65^{*}(1-65)\right]\right. \\
n=176
\end{gathered}
$$

Para la recolección de datos sociodemográficos se utilizó la ficha de Caracterización de la Persona con Enfermedad Crónica del Grupo de Cuidado de Enfermería al Paciente Crónico de la Universidad Nacional de Colombia (GCPC-UN) (18), mediante permiso escrito para su uso. La ficha de caracterización cuenta con tres dimensiones: 1. Identificación de condiciones del paciente y perfil sociodemográfico. 2. Percepción de carga y apoyo, y 3. Medios de información y comunicación. La ficha tiene un total de 22 ítems (18) y para su aplicación se debe contar con otros instrumentos anexos como son: la Escala de Valoración Funcional de PULSES y la prueba Short Portable Mental Status Questionnaire (SPMSQ), necesarias para medir las condiciones del paciente con DM2. Estos instrumentos hacen parte de la ficha de caracterización (GCPC-UN), cuyo proceso de validación y autoría es de la Universidad Nacional de Colombia y, como se describe al inicio de la metodología, se cuenta con la aprobación y autorización para su uso.

La Escala de Valoración Funcional de PULSES, creada y empleada por Moskowitz en 1957, consiste en un acrónimo inglés que se refiere a seis funciones así: $\mathrm{P}$ = estabilidad de la patología o condición física, $\mathrm{U}=$ utilización de miembros superiores, $\mathrm{L}=$ locomoción o función de los miembros inferiores, $\mathrm{S}=$ función sensorial, $\mathrm{E}=$ eliminación o control de esfínteres, $\mathrm{S}=$ capacidad de socializar (18). El perfil permite cuantificar, a través de una escala numérica, el nivel de funcionalidad (19). Este instrumento cuenta con una escala numérica de uno a cuatro, donde uno es independiente para la función, dos requiere de apoyo mecánico (aparato), tres requiere del apoyo mecánico y de otra persona, y cuatro es completamente dependiente en cada una de las funciones que son valoradas (20). Reporta una consistencia interna con una Alpha Cronbach de 0.74 (21).

El Short Portable Mental Status Questionnaire (SPMSQ) es un test de detección de deterioro cognitivo de aplicación muy rápida (5 minutos) que valora 4 parámetros: memoria a corto y largo plazo, orientación, información sobre hechos cotidianos y capacidad de cálculo, a través de 10 preguntas (20).

Este test se interpreta así: intacta (0-2 errores), alteración intelectual mínima ( 3 - 4 errores), alteración intelectual moderada (5 - 7 errores), la cual es considerada como patológica, y alteración intelectual severa (8 -10 errores) también patológica (22). Es importante aclarar que al momento del resultado se permite un error de más si la persona no ha recibido educación primaria (22). Por tanto, es útil en virtud de 
ISSN 1794-9831

E-ISSN 2322-7028

Vol. 14 No. 1

Ene - Jun 2017

Cúcuta, Colombia su fácil aplicación, no requiere materiales especiales para su administración y se puede aplicar a personas de bajos niveles de escolaridad (23).

La fiabilidad inter e intra observador del SPMSQ (versión en español) fue de 0,738 y 0,925, respectivamente, alcanzando la consistencia interna un valor de 0,82 . La validez convergente fue del 0,74 y la discriminación del 0,23. El área bajo la curva de la característica operativa del receptor (ROC) fue de 0,89 , siendo la sensibilidad y especificidad de 85,7 y 79,3 , respectivamente, para un punto de corte de 3 o más errores, observándose variaciones según nivel de escolarización y edad (20-23).

Teniendo en cuenta que sólo se describe el instrumento y no sus resultados, pues se trata de un estudio descriptivo y no de un estudio de intervención en los pacientes de acuerdo con las condiciones encontradas, se aclara que se contó con el apoyo de una psicóloga de la institución, por si se presentaba alguna crisis durante la entrevista.

Para la evaluación de la adherencia terapéutica se aplicó el instrumento resultado de enfermería Conducta Terapéutica: Enfermedad o Lesión 1609, creado por investigadores de la Universidad Industrial de Santander, empleado en personas con hipertensión arterial y/o diabetes mellitus tipo 2, La escala de medición es tipo Likert, donde 1 es nunca, 2 raramente, 3 en ocasiones, 4 frecuentemente y 5 constantemente, manifestado según el Nursing Outcomes Classification (NOC) (24).

La conducta terapéutica se define como acciones personales para paliar o eliminar patologías. Este instrumento de enfermería permite evaluar la adherencia a un tratamiento. Para su construcción, se consideró el cumplimiento de las recomendaciones terapéuticas dadas para el tratamiento específico de la hipertensión arterial, en personas con esta patología, teniendo en cuenta que el tratamiento terapéutico en éstas abarca tanto manejo farmacológico como no farmacológico (24). Los indicadores del resultado fueron operacionalizados por Orozco (25).

Los antecedentes del instrumento señalan aspectos psicométricos, como reproducibilidad y concordancia. Reportó un coeficiente de correlación intraclase (CCI) de 0.93 con IC95\%: 0.91 a 0.95 , es decir una reproducibilidad casi perfecta y, en cuanto a la segunda, se encontró un buen acuerdo dado por un promedio de diferencia de 0.016 y límites de acuerdo de Bland y Altman del $95 \%$ entre -0.54 y 0.55 (26).

Los investigadores de la Universidad Industrial de Santander, para una mejor aplicación y análisis del instrumento, realizan una versión mejorada a través de un estudio de diseño de evaluación de tecnologías diagnósticas. Los indicadores de la etiqueta de resultado fueron operacionalizados con base en el estudio previo y las recomendaciones de la literatura. De esta forma, se realizó análisis de la validez de constructo mediante la metodología Rasch, de acuerdo con Romero y Orozco (27), y se evaluó la reproducibilidad del instrumento en 100 personas.

El instrumento final, aplicado en esta investigación, del cual se obtuvo autorización por escrito de los autores, es una etiqueta unidimensional que incluye 11 indicadores, operacionalizados con una escala de 1 a 5 . Se precisa una confiabilidad de los ítems del 1.0. La varianza explicada $62 \%$, sin presencia de un segundo factor y no se presentó funcionamiento diferencial de los ítems por edad o sexo. La reproducibilidad fue de 0.98 (27).

Los datos obtenidos al finalizar la aplicación de los instrumentos, fueron validados a doble digitación y tabulados mediante la herramienta Epidata ${ }^{\circledR}$ 3.1, y analizados con software estadístico IBM SPSS Versión 22.

La presente investigación se cataloga como de riesgo mínimo, según Resolución No. 8430del 1993(28), debido a que no provocará ningún daño físico ni sufrimiento psicológico, pero puede conducir al participante a reflexionar sobre su comportamiento y recordar experiencias vividas. Se garantizó el cumplimento de los principios éticos: beneficencia, justicia y autonomía, los datos de identificación personales de los participantes obtenidos son confidenciales, se sometió el proyecto escrito a evaluación y se realizó presentación oral por la investigadora principal, recibiendo aprobación para la ejecución por parte del Comité de Ética de la institución de salud y de la Universidad Nacional de Colombia, según Aval-015-2016 del 18 de abril de 2016 en Acta 6.

\section{OBJETIVOS}

\section{Objetivo general}

Determinar la adherencia terapéutica en personas con diabetes mellitus tipo 2 a través de la etiqueta: conducta terapéutica: enfermedad o lesión presentes, 
que asisten a una institución de salud del Municipio

\section{Objetivos específicos}

- Describir la caracterización sociodemográfica, valoración funcional y mental presente en personas con DM2 que asisten a una institución de salud del Municipio de Bucaramanga.

- Describir la adherencia terapéutica a través de los componentes farmacológicos y no farmacológicos en personas con diabetes mellitus tipo 2 .

- Establecer la relación entre la adherencia terapéutica y tiempo de realización del diagnóstico de DM2.

\section{RESULTADOS}

La recolección de la información se realizó en el periodo comprendido entre los meses de abril y mayo del año 2016; las limitaciones se derivan del corto tiempo para que el investigador generara confianza en los participantes y permitiera establecer una relación que facilitara el reconocimiento propio de conductas para el control de la DM2.

\section{Caracterización sociodemográfica, valoración funcional y mental de la muestra de estudio}

El análisis de las variables sociodemográfica y condiciones de los participantes se realizó mediante técnicas de estadística descriptiva. A continuación se presenta la Tabla 1, en la cual se muestran las variables sociodemográficas del estudio y aspectos a considerar en las personas con diabetes, puesto que éstas influyen en las conductas orientadas hacia la salud y en la adherencia a tratamientos farmacológicos y no farmacológicos en las personas con DM2. 
Adherencia terapéutica en personas con diabetes mellitus tipo $2 \bullet$ Mayerli Katherine Rincón-Romero, Claudia Torres-Contreras, Katya Anyud Corredor-Pardo

ISSN 1794-9831

E-ISSN 2322-7028 Vol. 14 No. 1 Ene - Jun 2017 Cúcuta, Colombia
Tabla 1. Descripción de la Población (n=178).

\begin{tabular}{|c|c|c|}
\hline Característica Sociodemográfica & $\mathbf{n}$ & $\%$ \\
\hline Género Femenino & 112 & 62,9 \\
\hline Edad* & $67,2[65,7-68,8]$ & 10,45 \\
\hline \multicolumn{3}{|l|}{ Nivel de Escolaridad } \\
\hline Ninguno & 7 & 3,9 \\
\hline Primaria & 115 & 64,6 \\
\hline Secundaria & 44 & 24,7 \\
\hline Universidad & 10 & 5,6 \\
\hline Posgrado & 2 & 1,1 \\
\hline \multicolumn{3}{|l|}{ Estado Civil } \\
\hline Casado & 89 & 50,0 \\
\hline Viudo & 41 & 23,0 \\
\hline Soltero & 23 & 12,9 \\
\hline Separado & 13 & 7,3 \\
\hline Unión Libre & 12 & 6,7 \\
\hline \multicolumn{3}{|l|}{ Ocupación } \\
\hline Hogar & 111 & 62,4 \\
\hline Pensionado & 36 & 20,2 \\
\hline Empleado & 18 & 10,1 \\
\hline Trabajador Independiente & 13 & 7,3 \\
\hline Lugar de Residencia Urbana & 163 & 91,6 \\
\hline \multicolumn{3}{|l|}{ Estrato Socioeconómico } \\
\hline 1 & 34 & 19,1 \\
\hline 2 & 66 & 37,1 \\
\hline 3 & 47 & 26,4 \\
\hline 4 & 31 & 17,4 \\
\hline Religión Católica & 151 & 84,8 \\
\hline \multicolumn{3}{|l|}{ Compromiso Religioso } \\
\hline Alto & 104 & 58,4 \\
\hline Medio & 60 & 33,7 \\
\hline Bajo & 13 & 7,3 \\
\hline
\end{tabular}

Fuente: Ficha de Caracterización de la Persona con Enfermedad Crónica GCPC-UN-P, 2016. 
Se observan las características sociodemográficas valoradas por el estudio en los participantes con diagnóstico de diabetes mellitus tipo 2, donde la edad presentó una distribución normal (K-S 0,200), con un promedio de 67,2 años, con un intervalo de confianza de $95 \%$ entre 65,7 y 68,8 años. La mayor proporción $62,9 \%,(112)$ son mujeres, el nivel de escolaridad de los pacientes fue de $64,6 \%$ con primaria, el $24,7 \%$ con secundaria y los niveles de formación profesional con un $6,7 \%$, mientras que el $3,9 \%$ no tenía escolaridad. Sin embargo, todos los participantes sabían leer y escribir.

La distribución porcentual por estado civil muestra que el $50 \%$ de la muestra es casado y el 6,7\% está en unión libre; el 23\% de la población se encuentran viudo, el 7,3 \% separado y el $12,9 \%$ de la población es soltero.

La ocupación que predominó fue el hogar con $62,4 \%$, en concordancia con la mayoría del género femenino de la muestra, seguido de los pensionados con un $20,2 \%$. En cuanto al lugar de residencia, el 91,6\% vive en zona urbana. En la tabla 2 se evidencian las condiciones de estado mental, funcionalidad y años de diagnóstico de la DM2 de los participantes del estudio, cuya funcionalidad se valoró mediante el cuestionario de PULSES, encontrando que el 88,2\% de la muestra presentó baja disfunción.

Tabla 2. Condiciones de los participantes.

\begin{tabular}{lcc}
\hline \multicolumn{1}{c}{ Característica Clínica } & $\mathbf{n}$ & $\%$ \\
\hline Valoración Funcional & 157 & 88,2 \\
Baja Disfunción & 21 & 11,8 \\
Mediana Disfunción & 142 & \\
Deterioro Cognitivo & 36 & 79,8 \\
Intacta & 10 & 20,2 \\
Alteración Intelectual Mínima & & {$[5-15]$} \\
Años con DM2* & & \\
\hline datos describen mediana y RIQ
\end{tabular}

Fuente: Ficha de caracterización de la persona con enfermedad crónica GCPC-UN-P, 2016.

Con respecto al deterioro cognitivo, los participantes se mantuvieron sin alteración intelectual $(79.8 \%)$ y alteración intelectual mínima (20.2\%), lo cual garantizó el cumplimiento de los criterios de selección planteados en el estudio, que requería poder entender para diligenciar los instrumentos. En cuanto al tiempo con la enfermedad, expresado en años desde la realización del diagnóstico con DM2 (K-S 0,000), se observa que los datos analizados no presentan una distribución normal, con una mediana de 10 años y un rango intercuartil entre 5-15 años.

\section{Adherencia terapéutica y los componentes farmacológicos y no farmacológicos en personas con diabetes mellitus tipo 2}

El desarrollo de esta investigación demostró que el Instrumento para Medición de Adherencia Terapéutica en Personas con Diabetes basado en la etiqueta de resultado del NOC, en sus 11 indicadores, fue comprendido fácilmente por las personas reclutadas, siendo entendible, con un buen nivel de confiabilidad, lo cual lo hace adecuado para la evaluación de la adherencia terapéutica.

La determinación de la adherencia terapéutica se hizo teniendo en cuenta el promedio de los indicadores de adherencia según las puntuaciones dadas por los autores, de acuerdo con el instructivo del instrumento, para obtener una variable cuantitativa-continua. El resultado de la medición con la que se presenta la adherencia terapéutica de la muestra es una mediana de 3,7 y un rango intercuartil entre 3,3 - 4,1.

En la figura 1 se aprecia la distribución de la adherencia terapéutica, según las puntuaciones de los indicadores de resultado de acuerdo con la escala del NOC. 
ISSN 1794-9831

E-ISSN 2322-7028

Vol. 14 No. 1

Ene - Jun 2017

Cúcuta, Colombia
Figura 1. Distribución de datos según escala NOC- variable adherencia terapéutica.

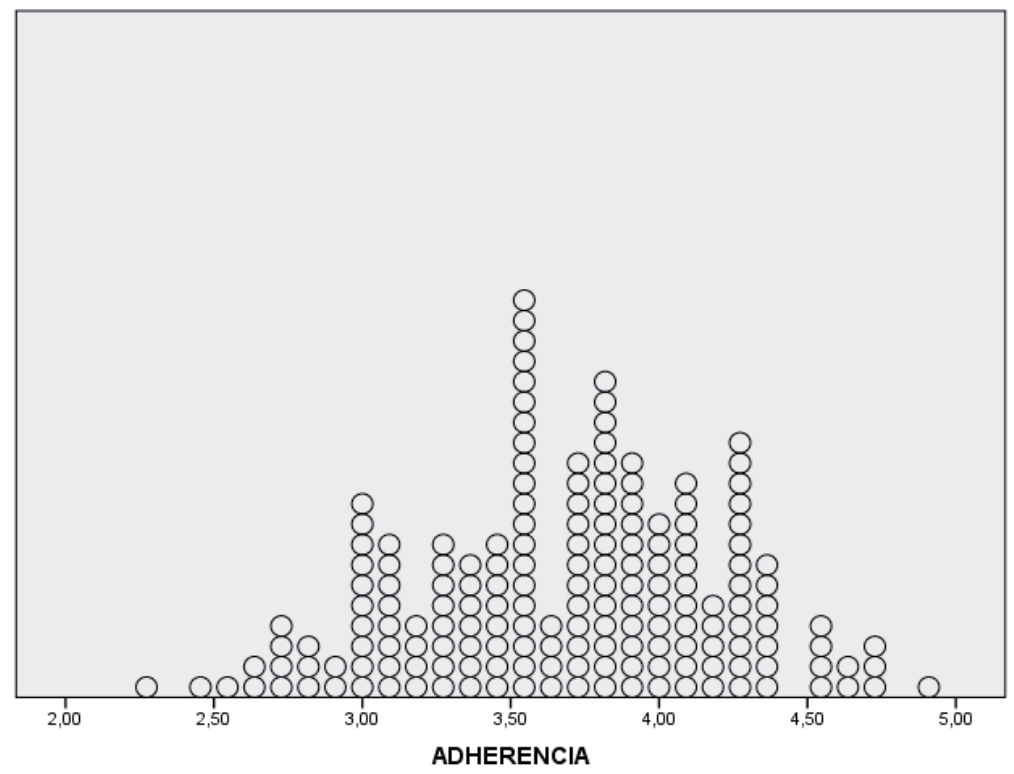

Fuente: Instrumento Conducta Terapéutica Enfermedad o Lesión 1609, 2016.

La Tabla 3 describe los componentes de la adherencia terapéutica de acuerdo con el análisis de los once indicadores del instrumento Conducta Terapéutica Enfermedad o Lesión, los cuales se miden en: constantemente manifestada, frecuentemente manifestada, en ocasiones manifestada, raramente manifestada y nunca manifestada, cuya escala de respuestas es 5, 4, 3, 2 y 1 , respectivamente (24). 
Tabla 3. Descripción por indicadores de adherencia terapéutica.

\begin{tabular}{|c|c|c|c|c|c|c|c|c|c|c|}
\hline \multirow{2}{*}{ Indicadores } & \multicolumn{2}{|c|}{ Nunca } & \multicolumn{2}{|c|}{ Raramente } & \multicolumn{2}{|c|}{ En Ocasiones } & \multicolumn{2}{|c|}{ Con Frecuencia } & \multicolumn{2}{|c|}{ Constantemente } \\
\hline & $\mathbf{n}$ & $\%$ & n & $\%$ & $\mathbf{n}$ & $\%$ & $\mathbf{n}$ & $\%$ & $\mathbf{n}$ & $\%$ \\
\hline Régimen de Medicación & 6 & 3,4 & 2 & 1,1 & 1 & 0,6 & 9 & 5,1 & 160 & 89,9 \\
\hline Actividades prescritas & 74 & 41,6 & 12 & 6,7 & 32 & 18,0 & 15 & 8,4 & 45 & 25,3 \\
\hline Dieta Prescrita & 1 & 0,6 & 3 & 1,7 & - & - & 46 & 25,8 & 128 & 71,9 \\
\hline Evita conductas que potencien la patología & 1 & 0,6 & 7 & 3,9 & 25 & 14,0 & 39 & 21,9 & 106 & 59,6 \\
\hline Solicita cita o asesoramiento profesional & 1 & 0,6 & 2 & 1,1 & 37 & 20,8 & 50 & 28,1 & 88 & 49,4 \\
\hline Supervisa los efectos terapéuticos & 18 & 10,1 & 38 & 21,3 & 27 & 15,2 & 31 & 17,4 & 64 & 36,0 \\
\hline Supervisa los cambios en el estado de enfermedad & 35 & 19,7 & 5 & 2,8 & 9 & 5,1 & 14 & 7,9 & 115 & 64,6 \\
\hline Supervisa los efectos secundarios del tratamiento & 115 & 64,6 & 14 & 7,9 & 10 & 5,6 & 12 & 6,7 & 27 & 15,2 \\
\hline Reconoce las complicaciones de ser diabético & 84 & 47,2 & 2 & 1,1 & 13 & 7,3 & 13 & 7,3 & 66 & 37,1 \\
\hline $\begin{array}{l}\text { Altera las funciones el rol para el cumplimiento } \\
\text { terapéutico }\end{array}$ & 8 & 4,5 & 51 & 28,7 & 55 & 30,9 & 42 & 23,6 & 22 & 12,4 \\
\hline Equilibra actividad y reposo & 1 & 0,6 & 2 & 1,1 & 15 & 8,4 & 73 & 41,0 & 87 & 48,9 \\
\hline
\end{tabular}

Ene - Jun 2017

Cúcuta, Colombia

Fuente: Instrumento Conducta Terapéutica Enfermedad o Lesión 1609, 2016.

El cumplimiento del régimen de la medicación mostró una adherencia en el indicador constantemente manifestada en el $89,9 \%$ de los participantes, con frecuentemente manifestada en el $5,1 \%$ y nunca manifestada en el 3,4\%. El nivel de cumplimiento de la actividad física prescrita se presenta con una adherencia con el indicador de nunca manifestada en el $41,6 \%, 25,3 \%$ en constantemente manifestada, $18 \%$ en ocasiones manifestada, $8,4 \%$ con frecuencia manifestada y $6,7 \%$ raramente manifestada.

Al abordar el seguimiento a la dieta prescrita, la adherencia para este indicador se encuentra en constantemente manifestada $71,6 \%$ de la muestra, el $25,8 \%$ con frecuencia manifestada, y niveles bajos de raramente manifestada $(1,7 \%)$ y nunca manifestada $(0,6 \%)$.

En el indicador de evita conductas que potencian su patología, la adherencia manifestada por la muestra estuvo en $59,6 \%$ como constantemente manifestada, el $21,9 \%$ en frecuentemente manifestada, el $14 \%$ en ocasiones manifestada, el $3,9 \%$ raramente manifestada y el $0,6 \%$ nunca manifestada.
En cuanto al indicador solicita cita con profesional sanitario cuando es necesario o requiere obtener asesoramiento de un profesional, se encontró una adherencia, constantemente manifestada en el $49,4 \%$ de la muestra, el $28,1 \%$ en frecuentemente manifestada, el $20,8 \%$ en ocasiones, el $1,1 \%$ raramente y el $0,6 \%$ nunca manifestada.

$\mathrm{El}$ indicador seis permite conocer el comportamiento de la persona con DM2 en relación con la supervisión de los efectos terapéuticos, con autocontrol glucémico, registro en casa de los valores obtenidos (fecha, hora y valores), así como la facilidad para conseguir un glucómetro y peso en el último mes. Al analizar este aspecto, se presenta una adherencia en constantemente manifestada para el $36 \%$, el $17,4 \%$ con frecuencia manifestada, el $15,2 \%$ en ocasiones manifestada, el $21,3 \%$ raramente manifestada y el $10,1 \%$ nunca manifestada.

Acerca de la supervisión de los cambios en el estado de enfermedad, este indicador evalúa al paciente en el reconocimiento de los síntomas de descompensación de la DM2. La adherencia de este indicador se 
ISSN 1794-9831

E-ISSN 2322-7028

Vol. 14 No. 1

Ene - Jun 2017

Cúcuta, Colombia encuentra en $64,6 \%$ para constantemente manifestado, el $19,7 \%$ de la muestra en nunca manifestado, el 7,9\% con frecuencia manifestado, el $5,1 \%$ en ocasiones manifestado y el 2,8\% raramente manifestado.

El comportamiento del indicador de supervisión de los efectos secundarios del tratamiento mostró una adherencia de $64,6 \%$ para el indicador nunca manifestado, el 15,2\% constantemente manifestado, el $19,7 \%$ nunca manifestado, el $7,9 \%$ con frecuencia manifestado, el $5,1 \%$ en ocasiones manifestado y el $2,8 \%$ raramente manifestado.

Los resultados respecto al indicador reconoce las complicaciones de su enfermedad y los síntomas asociados a ésta, muestran que el 47,2\% respondió nunca manifestado, el $37,1 \%$ constantemente manifestado, en ocasiones y con frecuencia manifestado presenta el $7,3 \%$ cada uno y raramente manifestado el $1,1 \%$.

Para evaluar las alteraciones de las funciones en el rol para el cumplimiento terapéutico, se examina si la persona durante el día interrumpe las actividades del hogar/trabajo para asumir conductas de salud y tratamiento, además de modificaciones de las funciones de los miembros de la familia para ayudarle al cumplimiento del tratamiento. La adherencia encontrada en la muestra para este indicador fue de $30,9 \%$ en ocasiones manifestada, de $28,7 \%$ con raramente manifestada, el $23,6 \%$ con frecuencia manifestada,el $12,4 \%$ constantemente manifestada y el $4,5 \%$ nunca manifestada.

El equilibrio entre actividad y reposo se interpreta como una adherencia al indicador de constantemente manifestada $(48,9 \%)$, con frecuencia $(41,0 \%)$, en ocasiones $(8,4 \%)$, raramente $(1,1 \%)$ y nunca manifestada $0,6 \%$ ).

\section{Relación entre la adherencia terapéutica y tiempo de realización del diagnóstico de DM2}

$\mathrm{Al}$ analizar la relación entre el tiempo de diagnóstico de la DM2 y la adherencia terapéutica a través de la prueba de correlación de Pearson, la figura 2 muestra que no se encontraron diferencias estadísticamente significativas evidenciadas por un valor de $\mathrm{p}$ de 0.39 y un $r$ de 0.064 , es decir, no existe un comportamiento diferencial de la variable tiempo con el diagnóstico y el riesgo de presentar o no adherencia al tratamiento. El comportamiento de la variable tiempo en años que lleva con la DM2 muestra un rango de 1-50 años, con una media de 11.5 años con la enfermedad crónica.

Figura 2. Relación entre años que lleva con la DM2 y adherencia terapéutica (Correlación de Pearson).

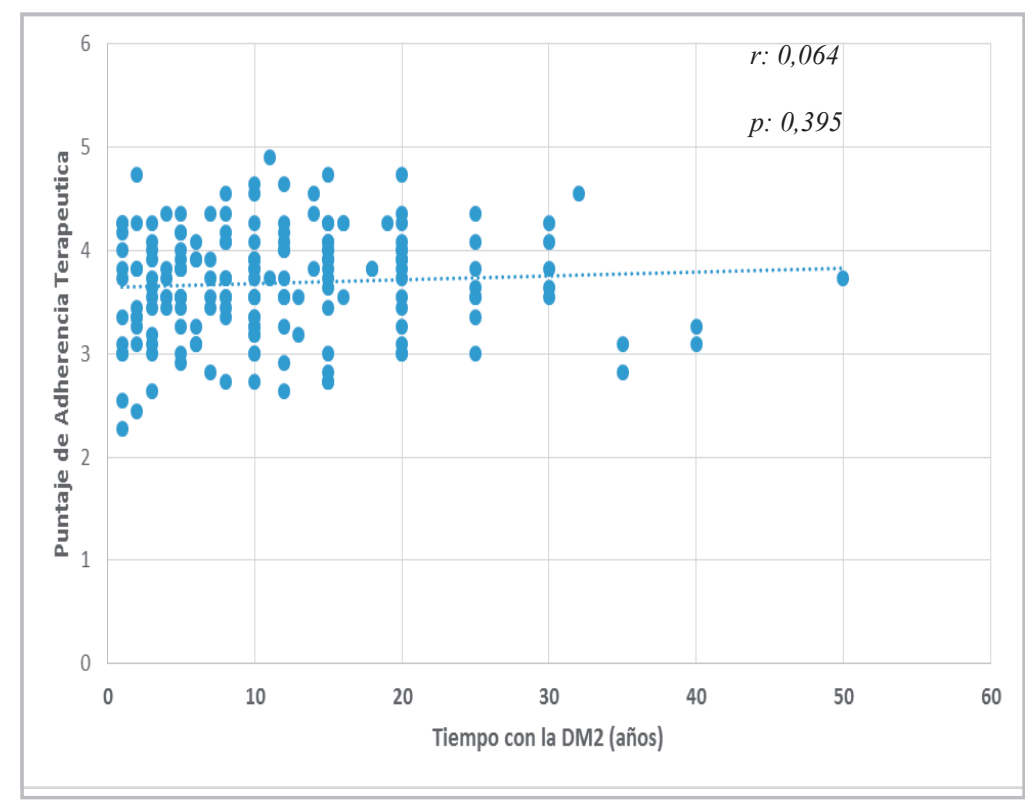

Fuente: Instrumento Conducta Terapéutica Enfermedad o Lesión 1609, 2016. 


\section{DISCUSIÓN}

Los datos sociodemográficos de este estudio reportaron una edad en la muestra promedio de 67,2 años, con un intervalo de confianza de $95 \%$ entre 65,7 y 68,8 años. Al respecto, datos preliminares del Observatorio de Salud Pública de Santander (OSPS) (7) identifican la prevalencia de esta enfermedad mayormente en personas de 45 a 64 años, y doble de frecuente en las mujeres en comparación con los hombres.

Figueroa y Gamarra (29) describen los factores asociados con no control metabólico en diabéticos pertenecientes a un programa de riesgo cardiovascular con una muestra mayoritariamente femenina $(54,3 \%)$ lo que concuerda con la muestra encontrada en el presente estudio. Es posible que la distribución por género de la muestra del estudio refleje mayor preocupación por el control de salud en las mujeres, en relación con la ocupación.

El nivel educativo se ha asociado con la prevención de las enfermedades crónicas, pues se indica una relación positiva entre este y la conducta preventiva (30). En el presente estudio predominó la educación primaria, seguida de la secundaria. A pesar de que se incluyeron personas sin ningún nivel educativo, se aclara que sabían leer y escribir, contrastando estos resultados con los descrito por Fagalde et al. (31) quienes refirieron que el nivel de escolaridad ha mostrado una relación inversa con la prevalencia de diabetes, lo que se traduce en un mayor promedio de factores de riesgo de DM2 en grupos con educación básica y media incompleta.

En este sentido, Barreto y Figueiredo (32) indican que los niveles básicos de educación proporcionan, en algún grado, fortalecimiento de relaciones personales, sociales e integración social, lo que aporta a la adherencia terapéutica. Esto se explica porque la educación es el resultado de procesos sociales de los individuos y puede contribuir significativamente a asumir conductas positivas hacia el cuidado de la salud.

Es de señalar que, a pesar de ser la institución donde se realizó el reclutamiento un centro de referencia de atención a población rural, el porcentaje de lugar de residencia en zona urbana correspondió al 91,6\%, lo que concuerda con lo señalado por Aschner (33) en su estudio, donde menciona que Colombia es un país en transición demográfica por el envejecimiento y la urbanización de su población; esto hace que la DM2 sea, por lo menos, cuatro veces más frecuente en la población urbana que en la rural, convirtiéndola en un factor de riesgo imparable, que se manifiesta en cambios del estilo de vida susceptibles de intervención tales como el sedentarismo y el deterioro de la alimentación que conducen a la obesidad, siendo estos contribuyentes para el desarrollo de DM2.

De otra parte, se ha descrito relación entre el estrato socioeconómico y la adherencia al tratamiento. Dávila et al. (34) encontraron asociación entre el bajo estrato socioeconómico y el abandono del tratamiento o el hecho de asumir estilos de vida saludable. En este estudio se encontró que la mayoría de los participantes son de estrato 2 y 3 , donde la mediana de adherencia al tratamiento fue de 3.7 .

Referente al tiempo con la enfermedad, expresado en años desde la realización del diagnóstico de DM2, se encontró una mediana de 10 años y un rango intercuartil entre 5-15 años. También, es similar a lo encontrado por Alba et al. (35) donde la mayoría de los pacientes estudiados fueron adultos mayores ( $>$ 65 años), lo que se relaciona con la historia natural de la diabetes mellitus tipo 2, de la misma forma que el tiempo promedio observado de evolución de la enfermedad fue de 13 años, lo que representa el carácter crónico de esta patología (36).

A pesar del carácter crónico de la DM2 y la complicaciones asociadas, la mayoría de personas incluidas en el estudio son funcionales, de acuerdo con los resultados de la valoración de PULSES (19), sumado a que la mayoría presenta la condición de no tener cuidador, lo que contribuye a indicar conservación de funcionalidad y autonomía.

Algunos autores (37-38) reportan que la funcionalidad reflejada en las actividades sociales, el sueño, el bienestar psicológico, la calma y los problemas físicos, entre otros, son ejemplos de aspectos que pueden afectar la calidad de vida del paciente, del cuidador y de la familia en general. Por ello, es importante conocer el grado de funcionalidad para cuidar al paciente $\mathrm{y}$, además, para prevenir el cansancio en el rol del cuidador que se asocia con la dependencia severa.

En cuanto al rol del cuidador, los hijos y el esposo (a) son los principales cuidadores, lo que coincide con
ISSN 1794-9831

E-ISSN 2322-7028

Vol. 14 No. 1

Ene - Jun 2017

Cúcuta, Colombia 
ISSN 1794-9831

E-ISSN 2322-7028

Vol. 14 No. 1

Ene - Jun 2017

Cúcuta, Colombia lo reportado por Gélvez et al. (39), quienes reportan el parentesco con los porcentajes más altos de tipos de cuidadores, donde se encuentra a los hijos de los pacientes representados en un $34,2 \%$, la madre del paciente $27,5 \%$ y el esposo (a) $22,5 \%$. La dedicación exclusiva a los cuidados por parte del cuidador implica una restricción de su vida social y, si a esto se suman situaciones de menor funcionalidad de la persona a quien se cuida, se experimenta mayor carga y sentimientos de depresión, lo cual termina repercutiendo en la persona con diabetes (40).

En lo concerniente al estado civil, el comportamiento de la muestra indica que la mayor proporción de la población se encuentra casada o en unión marital libre. Este hecho confirma que la composición familiar juega un papel fundamental en la adherencia terapéutica de las personas con DM2, convirtiendo el apoyo familiar en un elemento clave para el manejo y control de la enfermedad.

Rodríguez y Guerrero (41) consideran al apoyo familiar como un factor que influye en el control de la diabetes, aseverando que la participación familiar amortigua algunos efectos de los síntomas del paciente, en especial los psicológicos, e incide más sobre los comportamientos saludables que el apoyo social general. Para el presente estudio, la familia se identifica como la principal fuente de apoyo con un nivel de satisfacción alto, mientras que el apoyo psicológico fue el de más bajos niveles de satisfacción.

Zavala et al. (42) establecen en su estudio que los pacientes con DM no sólo son afectados físicamente, sino que también desarrollan problemas emocionales a medida que la enfermedad progresa, los cuales se manifiestan en miedo, incertidumbre, inseguridad y nerviosismo, entre otros.

Para Barrera et al. (43), en los programa de riesgo cardiovascular poco se ofrece el soporte de un profesional de psicología; por tanto, el llamado es a los profesionales de enfermería que lideran programas de promoción de la salud y prevención de la enfermedad, a ofrecer apoyo psicológico y establecer estrategia de remisión, de ser necesario, puesto que los apoyos con los que cuente la persona con DM2 determinan de manera contundente la forma en que vive la experiencia de la enfermedad crónica.

La prevalencia e incidencia de la DM2 en Colombia es una de las más grandes preocupaciones para los entes gubernamentales y las sociedades científicas. A pesar de los esfuerzos para el control y manejo de la diabetes, esta entidad clínica sigue constituyendo un importante problema de salud pública, lo que justifica la necesidad de intervenir de manera efectiva en el control de la adherencia terapéutica en la población afectada (1) y en la promoción de la salud y prevención de la enfermedad para evitar que aparezcan nuevos casos.

En el presente estudio la adherencia terapéutica fue de una mediana de 3,7 y un rango intercuartil entre $3,3-4,1$, es decir, una adherencia entre, en ocasiones manifestada: 3 a frecuentemente manifestada: 4. Al analizar el puntaje obtenido, de tal manera que un puntaje de 5 correspondió a adherencia constantemente manifestada y un puntaje de 1 correspondió a nunca manifestada, el puntaje promedio fue de 3,68 correspondiendo al $74 \%$ de la adherencia constantemente manifestada.

Al contrastar los hallazgos con reportes similares en el país, sobre la adherencia a los tratamientos en diabéticos tipo 2, Alayón et al. (13) en su investigación en la ciudad de Cartagena, señalan que obtuvieron un puntaje promedio de 4,6 , correspondiendo al $66 \%$ de la adherencia perfecta. De otro lado, la Asociación Americana de Endocrinólogos Clínicos (AACE) (44) reportó en el período 2003-2004 (cerca del 70\%) y el informe de la OMS (5) acerca de la adherencia a los tratamientos de larga duración, plantea que "en los países desarrollados, la adherencia a los tratamientos a largo plazo en la población general es de alrededor de $50 \%$ ".

Autores como González et al. (45), ofrecen cifras poco alentadoras respecto a la adherencia al tratamiento, donde el $78.3 \%$ de las personas estudiadas se ubicó como no adherentes a su tratamiento, similar a lo encontrado en Bucaramanga por Figueroa y Gamarra (29), donde la prevalencia de pacientes no adherentes al tratamiento fue de $65,6 \%$, datos que difieren de los analizados para este estudio .

Con el fin de abordar el fenómeno de adherencia terapéutica de una forma más completa, se realizó un análisis de los indicadores que componen la adherencia terapéutica, encontrándose mayores porcentajes de adherencia constantemente manifestada en los indicadores: cumple con el régimen de la medicación, dieta prescrita, supervisa los cambios en el estado de la enfermedad y evita 
conducta que potencian la patología (tabaquismo, consumo de alcohol y estrés). Lo contrario se describe en los indicadores: cumple actividades prescritas, supervisa los efectos secundarios del tratamiento y reconoce las complicaciones de ser diabético los cuales presentaron niveles de adherencia terapéutica nunca manifestada por encima del $40 \%$.

Al respecto, Alayón et al.(13) en su estudio, encontraron en su estudio que los puntajes más elevados se registraron para la ausencia del hábito de fumar (94.2\%) y el cumplimiento de medicación $(88.5 \%)$, mientras que las peores puntuaciones se registraron en el automonitoreo de glucosa, la práctica de ejercicio físico y las actividades preventivas como cuidado de extremidades. A esto se suma lo reportado por la OMS (5), que señala que es importante evaluar el grado de la adherencia a cada componente del régimen de tratamiento en forma independiente, en relación con el régimen de medicación que reporta cifras de adherencia entre 70 y $80 \%$.

El fenómeno de la adherencia terapéutica supone retos para el profesional de enfermería, dado que permite orientar el actuar de enfermería y diseñar e implementar estrategias de intervención para el cuidado de la salud cardiovascular de las personas con diabetes tipo 2, además de dar solución a los problemas planteados en la práctica diaria, asociados a los comportamientos de adherencia terapéutica y a sus factores influyentes.

\section{CONCLUSIONES}

Los resultados indicaron que existe una prevalencia mayor de diabetes mellitus 2 en mujeres que en hombres. A pesar de que la media de edad fue de 67,2 años, en relación con la mediana de 10 años de vivencia de la enfermedad crónica, deja al descubierto una afectación de DM2 que se da entre la cuarta y quinta década de vida, etapa aún productiva y laboral en el ser humano, y a enfermería le corresponde tomar parte en el proceso de atención mediante el diseño e implementación de estrategias que contribuyan a disminuir las complicaciones derivadas del inapropiado control de la DM2. El control de la DM2 es importante para la salud pública no sólo porque afecta a personas productivas sino porque, con el incremento de la esperanza de vida al nacer, los retos para tener una vida saludable con esta enfermedad crónica son aún mayores.
Las personas con diagnóstico de DM2 del estudio presentan una adherencia terapéutica según los indicadores de resultado del NOC (22) entre: en ocasiones manifestada y con frecuencia manifestada, con una media de 3,68. El incumplimiento de actividad física prescrita fue el factor que presentó mayores cifras de no adherencia. Teniendo en cuenta esta información, se precisa generar estrategias que vinculen los factores con adherencia nunca, raramente y ocasiones manifestada, para generar beneficios a corto, mediano o largo plazo, según sea la capacidad de incluir dentro de la rutina el hábito del ejercicio, junto con la dieta y demás indicaciones sugeridas en el tratamiento.

No se encontraron relaciones estadísticamente significativas entre la adherencia terapéutica y los años de diagnóstico de la DM2. Por tanto, las intervenciones o estrategias deben estar diseñadas para brindar un soporte durante todo el curso de la enfermedad. Una adhesión inicial no garantiza la continuidad en la adhesión, siendo muy probable que esta disminuya a medida que transcurre el tiempo y que los pacientes que siguen o se adhieren a un aspecto de su programa de tratamiento, a menudo, se comportan de manera muy diferente con otras demandas de su programa.

La adhesión debe contemplarse como un fenómeno complejo y dinámico, dependiente de muchas variables y susceptible de cambiar con el paso del tiempo. La adherencia del paciente a un rasgo del régimen terapéutico no asegura su adhesión a los otros rasgos del mismo ni al mismo rasgo en otro momento del tiempo, y es, por ende, difícil de abordar tanto desde el punto de vista teórico e investigativo como práctico.

Para futuras investigaciones se plantea la necesidad de controlar los aspectos sociodemográficos para la medición estadística, con el fin de analizar y describir factores influyentes en la adherencia terapéutica; por tanto, se sugiere equiparar la muestra por género, establecer grupos de edad, comorbilidades, características de accesibilidad a servicios de salud y utilizar la hemoglobina glucosilada (HbA1c) que, para este estudio no se pudo reportar por limitaciones económicas. Esto dará mayor validez a los resultados, en contraste con lo reportado por el paciente sobre la realidad del nivel de control y compensación metabólica de la DM2. 

Anyud Corredor-Pardo

ISSN 1794-9831 E-ISSN 2322-7028 Vol. 14 No. 1 Ene - Jun 2017 Cúcuta, Colombia

\section{CONFLICTO DE INTERESES}

Las autoras declaran no tener ningún conflicto de intereses. 


\section{REFERENCIAS BIBLIOGRÀFICAS}

1. González JC, Walker JH, Einarson TR. Diabetes mellitus tipo 2 en Colombia: costo de la enfermedad. Rev Panam Salud Pública. 2009; 26: 55-63.

2. Alpízar-Salazar M. Guía para el manejo integral del paciente diabético. México: El Manual Moderno; 2001.

3. Zimmet P, Shaw J, Murria S, Sicree R. La epidemia de diabetes en crecimiento: predecir el futuro. Diabetes Voice 2003; 48(número especial): 12-16.

4. Del Valle M. Epidemiología de la diabetes. XIX Congreso Latinoamericano de Patología Clínica/ML. La Habana: Asociación Latinoamericana de Patologías Clínicas -ALAPAC; 2009. p. 1-54.

5. Organización Mundial de la Salud (OMS). Adherencia a los tratamientos a largo plazo: pruebas para la acción. Washington, D.C. OMS; 2004.

6. O’Donnell CJ, Elosua R. Factores de riesgo cardiovascular. Perspectivas derivadas del Framingham Heart Study. Rev Esp Cardiol. 2008; 61(3): 299-310.

7. Observatorio de Salud Pública de Santander (OSPS). La diabetes: una enfermedad cada vez más frecuente. Actualidad OSPS .[Internet]. 2016 [consultado 18 de mayo de 2016]. Disponible en: http://web.observatorio. co/la-diabetes-una-enfermedad-cada-vez-mas-frecuente-especialmente-en-las-mujeres-y-adultos-mayores-de$\underline{45-\text { anos }}$

8. Hidalgo C, Carrasco E. Salud familiar: Un modelo de atención integral en la atención primaria. Santiago: Ediciones Universidad Católica Chile; 1999.

9. Federation Internacional Pharmaceutical-FIP. Declaración de las normas profesionales de FIP: El papel del farmacéutico en la estimulación de la adherencia a los tratamientos a largo plazo. Approved by FIP Council in Sydney in September 2003.

10. Gonzáles-Castro P. El apoyo familiar en la adherencia al tratamiento nutricional del paciente con diabetes mellitus tipo 2 (DM2). Revista Waxapa. 2011; 3(5): 102-7.

11. International Diabetes Federation (IDF). IDF Diabetes Atlas. 7th ed. [Internet]. Brussels, Belgium; IDF; 2015 [consultado 13 de enero de 2016]. Disponible en: www.diabetesatlas.org

12. Álvarez-Mabán E, Barra-Almagiá E. Autoeficacia, estrés percibido y adherencia terapéutica en pacientes hemodializados. Cienc. enferm. [Internet]. 2010 [consultado 13 de enero de 2016]; XVI (3): 63-72. Disponible en: http://www.scielo.cl/scielo.php?script=sci arttext\&pid=S0717-95532010000300008\&lng=es. http://dx.doi. org/10.4067/S0717-95532010000300008.

13. Alayón AN, Mosquera-Vásquez M. Adherencia al Tratamiento basado en Comportamientos en Pacientes Diabéticos Cartagena de Indias, Colombia. Rev. Salud pública [Internet]. 2008 [consultado 23 de enero de 2016]; 10(5): 777-787. Disponible en: http://www.scielosp.org/scielo.php?script=sci arttext\&pid=S012400642008000500010\&lng=en. http://dx.doi.org/10.1590/S0124-00642008000500010

14. Tamayo DC. Diabetes en Colombia. Experiencias en programas y modelos de atención. Observatorio de Diabetes de Colombia. [Internet]. Colombia: Observatorio de Diabetes de Colombia; 2012 [consultado 18 de julio de 2016]. Disponible en: http://www.odc.org.co/files/Diabetes_en_Colombia_experiencias_en_programas_y modelos de atencion.pdf

15. Otero L, Muñoz A, Figueroa L. Impacto del Modelo Moderador del Riesgo en la Enfermedad Renal Crónica. IPS COMFANDI, Cali, 2006. Colomb Med. 2007; 38(3): 274-81.

16. Gómez DA, Mahecha M, Gómez RD, Duque JE, Agudelo SM. Características de la Diabetes Mellitus en una red de servicios de primer nivel de atención. Medellín, 2005-2008. Rev Fac Nac Salud Pública. 2011; 29(1):7-17.

17. Colombia Ministerio de la Protección Social, Programa de Apoyo a la Reforma de Salud (PARS). Guía 17. Guía de atención de la diabetes mellitus tipo II. En: Minprotecciónsocial, PARS: Guías de promoción de la salud y prevención de enfermedades en la salud pública. Bogotá: Minprotecciónsocial; 2007. p. 362-439.

18. Ortiz LB, Rosero EV, Cendales PA. Encuesta de caracterización para el cuidado de una persona con enfermedad crónica. Investig. Enferm. Imagen Desarr. 2014; 17(1).

19. Granger CV, Albrecht GL, Hamilton BB. Outcome of Comprehensive Medical Rehabilitation messurement by PULSES profile and the Barthel Index. Arch Physmed Rehabil. 1979; 60(4): 145-54.

20. Venegas-Bustos BC. Habilidad del cuidador y funcionalidad de la persona cuidada. Aquichan 2006; 6(1): 13747.

21. Marshall SC, Heisel B, Grinnell D. Validity of the PULSES profile compared with the Functional Independence Measure for measuring disability in a stroke rehabilitation setting. Arch Phys Med Rehabil. 1999; 80(7): 760-5.

22. Pfeiffer E. A short portable mental status questionnaire for the assessment of organic brain deficit in elderly patients. J Am Geriatr Soc. 1975; 23(10): 433-41.

23. Martinez-Iglesia J, Dueñas-Herrero R, Onís-Vilches MC, Aguado-Taberné C, Albert-Colomer C, Luque- Luque R. Adaptación y validación al castellano del cuestionario de Pfeiffer (SPMSQ) para detectar la existencia de 
deterioro cognitivo en personas mayores e 65 años. Med Clínica; 2001; 117(4): 129-34.

24. Moorhead S, Johnson M, Maas ML, Swanson E. Clasificación de Resultados de Enfermería (NOC). $4^{\mathrm{a}}$ ed. Barcelona: Elsevier; 2009.

25. Orozco LC , Camargo F. Operacionalización del resultado de enfermería conducta terapéutica: enfermedad o lesión. [Tesis Postgrado]. Bucaramanga: Universidad Industrial de Santander; 2010.

26. Orozco LC. Medición en salud, diagnóstico, evaluación de resultados. Un manual critico mas alla de lo basico. $1^{\mathrm{a}}$ ed. Bucaramanga: Publicaciones UIS; 2010.

27. Romero S, Orozco L. Validación de la etiqueta de resultado de enfermería: conducta terapéutica enfermedad o lesión. En: Memorias [Internet]. XIV Coloquio Panamericano de investigación en Enfermería; 2014 [consultado 18 de mayo de 2016]: p. 484. Disponible en: http://acofaen.org.co/wp-content/uploads/2014/11/memoriascoloquio_af final.pdf

28. República de Colombia, Ministerio de Salud . Resolucion 8430 de 1993, 0ctubre 4, por la cual se establecen las normas cientifico tecnicas y administrativas para la investigacion en salud. Bogotá D.C.: Ministerio de Salud; 1993.

29. Figueroa CM, Gamarra G. Factores asociados con no control metabólico en diabéticos pertenecientes a un programa de riesgo cardiovascular. Acta Med Colomb [Internet]. 2013 [consultado 23 de enero de 2016]; 38(4): 213-221. Disponible en: http://www.scielo.org.co/scielo.php?script=sci arttext\&pid=S012024482013000400006\&lng=en

30. Lucumí-Cuesta DI, Gómez-Gutiérrez LF. Accesibilidad a los servicios de salud en la práctica de citología reciente de cuello uterino en una zona urbana de Colombia. Rev. Esp. Salud Pública [Internet]. 2004 [consultado 14 de enero de 2016]; 78(3): 367-377. Disponible en: http://scielo.isciii.es/scielo.php?script=sci_arttext\&pid=S113557272004000300006\&lng=es

31. Fagalde MP, Del Solar JA, Guerrero M, Atalah E. Factores de riesgo de enfermedades crónicas no transmisibles en funcionarios de una empresa de servicios financieros de la Región Metropolitana. Rev Med Chile 2005; 133(8): 919-28.

32. Barreto SM, Figueiredo R. Doença crônica, auto-avaliação de saúde e comportamento de risco: diferença de gênero. Rev Saùde Pública 2009; 43(Supl 2): 38-47.

33. Aschner P. Epidemiología de la diabetes en Colombia. Av Diabetol. 2010; 26(2): 95-100.

34. Dávila CA, Agudelo M, Hernández LEG. Diabetes en México y Colombia: Análisis de la tendencia de años de vida perdidos, 1998-2007. Rev. salud pública [Internet]. 2011 [consultado 30 de enero de 2016]; 13(4): 560-571. Disponible en: http://www.scielo.org.co/scielo.php?script=sci_arttext\&pid=S0124$00642011000400002 \& \operatorname{lng}=$ en.

35. Alba LH, Bastidas C, Vivas JM, Gil F. Prevalencia de control glucémico y factores relacionados en pacientes con diabetes mellitus tipo 2 del Hospital Universitario de San Ignacio, Bogotá, Colombia. Gac Méd Méx. 2009; 145(6): 469-74.

36. Asociación Latinoamericana de Diabetes. Guías ALAD de diagnóstico, control y tratamiento de la Diabetes Mellitus Tipo 2. Rev Asoc Latinoam Diabetes. 2006;14: 111-6.

37. Rodríguez-Sánchez E, Mora-Simón S, Porras-Santos N, Patino-Alonso MC, Recio-Rodríguez JI, BecerroMuñoz C, et al. Effectiveness of an intervention in groups of family caregivers of dependent patients for their application in primary health centers. Study protocol. BMC Public Health. 2010; 10(1): 559.

38. Flores E, Rivas E, Seguel F. Nivel de sobrecarga en el desempeño del rol del cuidador familiar de adulto mayor con dependencia severa. Cienc Enferm. 2012;18(1): 29-41

39. Gélvez-Martínez JL, Arias-Hoyos SL, Cárdenas-Mosquera VC. Habilidad del cuidador de pacientes con patología crónica discapacitante en casa. Rev Médica Sanitas; [Internet]. 2014 [consultado 30 de enero de 2016]; 13(4): 560-571. Disponible en: http://www.unisanitas.edu.co/Revista/50/Habilidad del_Cuidador de Pacientes.pdf

40. Barrera L, Pinto N, Sánchez B, Carrillo G, Chaparro L. Cuidando a los cuidadores: familiares de personas con enfermedad crónica. $1^{a}$ ed. Bogotá D.C.: Universidad Nacional de Colombia, Facultad de Enfermería; 2010.

41. Rodríguez-Morán M, Guerrero-Romero JF. Importancia del apoyo familiar en el control de la glucemia. Salud pública Méx [Internet]. 1997 [consultado 3 de mayo de 2016]; 39(1): 44-47. Disponible en: http://www.scielosp. org/scielo.php?script=sci_arttext\&pid=S0036-36341997000100007\&lng=en. http://dx.doi.org/10.1590/S0036$\underline{36341997000100007}$

42. Zavala M, Vázquez $\mathrm{O}$, Whetsell, $\mathrm{M}$. Bienestar espiritual y ansiedad en pacientes diabéticos. Rev Aquichan, 2009. [consultado 23 de enero de 2016]; 6(1): 8-21. Disponible en: http://aquichan.unisabana.edu.co/index.php/ aquichan/article/view/76

43. Barrera-Ortiz L, Vargas-Rosero E, Cendales PA. Encuesta de caracterización para el cuidado de una persona con enfermedad crónica. Investigación en Enfermería: Imagen y Desarrollo. 2015 [consultado 23 de enero de 2016]; 17(1): 27-43. Disponible en: http://revistas.javeriana.edu.co/index.php/imagenydesarrollo/article/ 
view/9122/11922 doi:http://dx.doi.org/10.11144/Javeriana.IE17-1.eccp.

44. Asociación Americana de Endocrinólogos Clínicos (AACE). Directrices médicas para el tratamiento de la diabetes mellitus: El sistema AACE de autotratamiento intensivo de la diabetes. Endoc Pract. 2005; 8(Suppl 1): 40-82.

45. González JO, González MA, Vázquez J, Galán S. Autoeficacia y adherencia terapéutica en personas con diabetes mellitus tipo 2. Revista de Investigación en Psicología [Internet]. 2015 [consultado 23 de junio de 2016]; 18(1): 47-61. Disponible en: http://revistasinvestigacion.unmsm.edu.pe/index.php/psico/article/view/11772 